horizontale où les vapeurs de zinc se convertissent en fumćes d'oxyde. De là, ces fumées sont aspirées dans les chambres de dépôt, adossćes au mur devant lequel on voit le four. Les charges et les coulées ont lieu toutes les deux heures et dans l'intervalle de ces opérations qui se suivent, aucune fumée ne se dégage des apparels ainsi qu'en témoigne la photographie.

Un seul manoumre suffit à conduire le four, aidé seulement pendant quelques minutes, par un autre manouvre au moment de la charge, qui est très rapide, et dure par elle-même moins de deux minutes; les coulées se font en 3 ou 4 minutes au plus. Deux hommes pourraient donc conduire très aisément deux fours de beaucoup plus grande capacité. Une fois les appareils réglés pour un minerai de nature donnée, ce qui se fait en moins de 24 heures, ils marchent alors des semaines entières sans autre maind'ouvre que celle dont ll vient d'être question, la seule condition exigée des ouvriers étant de répéter bien ponctuellement toujours les mêmes machinales opérations.

Il est à noter que tous les essais de l'usine d'Arudy ont été effectuées exclusivement avec des ouvriers pris dans le pays même, cultivateurs, bûcherons ou carriers, qui n'avaient jamais vu le moindre four métallurgique; leurs salaires varient de $2 \mathrm{fr} .75$ à $3 \mathrm{fr} .25$. C'est dire combien le four électrique est un appareil plus facile à conduire que les fours belges et silésiens. - Etant donné que la fabrication de l'oxyde est presque aussi délicate que l'obtention du zine métalique, on est fondé à croire que la même maind'œuvre, une fois un peu mieux exercée, produirait également bien le métal.

Dans les comptes rendus, d'une série d'essais du procédé au cours desquels on a fondu un peu plus de 76 tonnes de minerai avec des consommations d'énergie très sensiblement égales à celles des essais précédemment cités, nous avons relevé les chiffres suivants :

En 600 heures de marche, soit 25 jours, on a fondu 28345 kilos de blende à gangue de silice à peu près pure, contenant de 2 à $4 \%$ de fer, $1 \%$ de métaux étrangers et titrant en moyenne $37,28 \%$ de zinc.

Le courant débité pendant ce temps était de $3800 \mathrm{am}$. pères sous 42 volts avec $\cos \frac{\pi}{2}=0,85$.

La quantité de chaux correspondante enployée, tant comme réactif que comme fondant, a ćté de $1238+$ kilos, et la quantité de charbon réducteur de 3 i 46 kilos.

Le poids de zinc contenu dans le minerai passé au four était de 10587 kilos, devant théoriquement donner 13 i $93 \mathrm{k}$. d'oxyde. On a récolté effectivement que i2 370 kilos.

La teneur en zinc des laitiers et du sulfure de calcium a varié pendant ces essais de I, 2 à $1,8 \%$.

Il a été em ployé 1286 kilos d'électrodes, mais de ce poids il faut défalquer 6.7 kilos représentant les parties non brûlées, hors du four, et que, dans une usine mieux concue l'on pourrait utiliser plus completement.

Le garnissage du four doit être refait après deux mois de marche, par suite des déformations qu'il subit.

Ftant tenu compte des mêmes éléments de prix de revient que précédemment, auxquels il faut ajouter le coke payé $30 \mathrm{fr}$. la tonne à l'usine, les 12370 kilos d'oyyde de la série d'essais qui vient d'être indiquée ont couté $+598 \mathrm{fr}$., sur wagon a l'usine. 8500 kilos ont été vendu, $49 \mathrm{fr} .60$ les 100 kilos et 3870 kilos, 48 fr. 75 au départ d'Arudy, ce qui représente une recette totale de $6 \mathrm{1}_{2} \mathrm{fr}, 60$.

Ceci montre quels bénéfices ont pourrait réaliser dans une usine de 1200 à 1500 chevaux, payant le cheval $45 \mathrm{fr}$. et non plus $65 \mathrm{fr}$. - puis fabricant ses électrodes.
L'oxyde de zinc obtenu se classe en deux catégories suivant sa blancheur; la plus grande partie: $70 \%$ environ constitue la meilleure qualité ; 3 à $4 \%$, formés des parties recueillies dans les cheminées allant aux chambres, sont repassés avec les charges. Les deux qualités ont très sensiblement le même degré de pureté; elles titrent 98,6 à $99 \%$ d'oxyde de zinc; $0,4 \%$ d'humidité; 0,2 à $0,3 \%$ de fer et 0,5 a $0,7 \%$ de silice, chaux et métaux étrangers, ces derniers à l'état de traces seulement, quand les minerais eux-mêmes en contiennent pen.

Sur la quantité produite à Arudy, trois cents et quelques fûts de roo kilos ont été vendus à Marseille, à Paris et à Lyon, où ils ont été cotés aux cours des oxydes de prove. nance allemande et américaine qui sont fabriqués par les anciennes méthodes

Les chifres des résultats d'essais précédemment relatés, sont bien moins séduisants que ceux publiés sur la mème question par des électrométallurgistes en renom. Sans doute ce fait est dù à une insuffisamment longue connaissance du four électrique chez les expérimentateurs qui nous occupent, à leur manque de pratique en matiere de métallurgie du zinc. Mais peut-être cette infériorité non contestable, tient elle aussi au fait que les résultats obtenus par MM. Côte et Pierron, ressortent de chiflres pris sur des fours en marche à peu pres industrielle et soumise à tous les aléas dpo débuts, chiffres sincères et non calculés d'après quelques données d'expériences heureuses en laboratoire. La Société des Fonderies Electriques met d'ailleurs son usine d'Arudy à la disposition de toute Société miniere que la démonstration de ces résultats peut intéresser, et cela en lui oflrant de fondre 20 à 30000 kilos de son miminerai, dans les fours prêts à le recevolr.

Conclusions, - On peut en toute sécurité conclure que l'obtention du blanc de zinc commercial par traitement de minerais crûs à plus ou moins faible teneur au four électrique, est économiquement possible avec du cheval-an au prix de 40 a $50 \mathrm{fr}$. Bien des mines, que leur position éloignée des voies de transport ne permet pas d'exploiter avantageusement, peuvent d'abord trouver dans des procédés analogues, trìs améliorables, un nouvel élément de vitalité. C'est la deuxième étape de la marche entreprise par MM. Côte et Pierron, et qu'ils se proposent maintenant d'aborder. Puis, la première fonderie qui fera du blanc de zinc sur une échelle réellement industrielle pendant quelques années, sera vite maitresse de l'obtention du métal, avec de bons rendements. Alors la solution du probleme pourra se généraliser.

Eugène Fleurvitif:

\section{La Fabrication des Électrodes}

(siuile)

\section{II. - MATIÈRES PREMIÈRES EMPLOYÉES DANS LA FABRICATION DES ÉLECTRODES}

Ginevalibs. - Les malieves premieres de la fomicalion dos clectrodes sont les charbons et los agolomémnts.

Les charbons omployes sont maturols ou artildcicls. Co sont : l'anthracite, le charluon de comue, lo coke de vetrole. le coke de hrai, auxquels il fath adjoudre, mais seulement a titre secondaire, lo graphite naturel, to graphite aplificiel, le noir de fumoe, la houille grasse bien collante et le char. bon de bois. 
les atgolomónuts employis sont les goudrous de tis/illation pyrogénée de la lioullle; le brai, rócidu de distıllatron des goudrons de houille; les goudrons synthrluques. obtenus en (lissolvant du brai dans les hules de goudron de houmle. $\Lambda$ ces corps, il laut ajouter le bitume et l'asqhalle, qui sont donés d'un pouvoir aggloncrant concidérable.

$I$ - Charbons. - Nous étudiorons : 1" los charjums nalurels et 2" les charbons artificiels.

10 Churbons nuturels.-- Les cliarbons naturels employéi la talmricalion des électrodes sont : a) L'anthacite, bj la houille, $c$ ) le graphite.

(c) Anthracitr. - L'anthracite, appelée également hourlle élatante et charbon incombustible, hrile avec difficulte et ne donne qu'une flamme très counte, sans fumée ni odeur, mais dégage, en hrulant, beaucoup de chalem.

I intmacile est un copps completement dépourvir de forme matalline, ce yu le distingue du craphite. Il est d'un beau noil brillant, a cassure conchotdale sonvent Irrare, sècho au toucher, avec un éclat métallifue assez prononcé. Il est composí de 90 a $91 \%$ do callone, el ne contient qu'une tris petite quantits de matries volalilos

L'anthracite, qui forme une variéti de houlle hich caractéristupe, paraît rependant poséeler me conshlution phystque of ehimaqu nettement differonte de celle de la houlle juroprement dite.

Lo véritable anthracile ne tacho pas les doigt- romme la houlle. Il ne prosento ni los crévastes le la houlle millante, ni los stratifirations le la houille mate c"est, de toms les challons fosstes, celini qui a le moins conserve les raratires de son origine végétale; rependant, on conslate, on l'incincrant, des traces de structure stratifiée.

L'anthracite résiste trés énergiquement a l'action de prosque tous los ruactuls rhimiques, auss l'ctude de sa constitution est-olle fort dulfele. Cependant, lorsqu'il est rn osifulles minces, l'acule sulfurique concentré et boullant l'atlarue on se colorant en brun rougeatre ces esrubles deviement alors translucides sur les bords, mais on $n y$ pout recomait.e aucune trace dorgancation. En fai-ant agir a chaud le chlorate de potassium avec l'achde nutrique concentré, on est parvenu, sur des echantillons d'anthriole de Pensylvanie, it reconnaitre, apres traitement par l'alcool, des tissus végélaux à structuro très note, en par- surtout entre le schiste algileux el lo schiste micacé.

in France, on trouve de lanthracite dans la Loire, la Sarthe ef la Mayenne. lin Ventée cxiste un bassin de 20 hlomètes de long sur un hilonetre de lare. Dans le bassin d'Alals se trouvent de minces rouches d'anthracite. Cilons encore dans l'abe le bassin de Ségure, (qui est à découvert sur une longucur de 4 kilomètres, et les wiscments de Castanet et de St-Geniès, dans l'Hérault,

Dans l'Isère 11 existe, à la Mure, un important gisement d'anthracite Dans un grand nombre de régions alpestros on rencontre également ce combustible, principalement $\mathrm{cn}$ Ilaute-Savoie.

Parmi les pays de l'burope qui ont des mines d'anthracile, il nous faut signaler l'Angletere (Paye de Galles ot rogion d'lidimhourg), ia Suicse (Valais) et la Russie (rigmon du lac Onéga).

En Amérique, on rencontre l'anthracite en Pensylvanio; on trouve encore dimportants gisements au Chrli et clans l'extrémité sud de la Patagonje.

En Acie, la Chine possede uno vaste rusorvo d'anthracite.

Cr.jont foredefuns exemples d'analyses danthracite effertuces par divers auteurs.

\begin{tabular}{|c|c|c|c|}
\hline 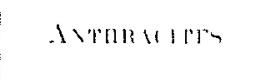 & $\left.C x^{3}\right)$ गय & 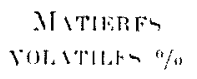 & SOUI IE $\%$ \\
\hline 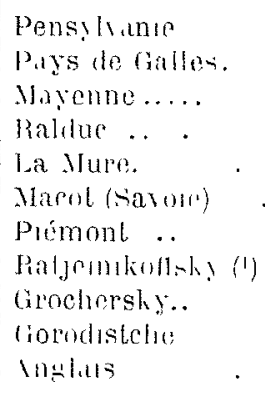 & 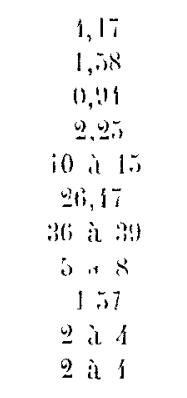 & $\begin{array}{c}- \\
- \\
- \\
- \\
- \\
10 \text { ì } 13 \\
- \\
- \\
\text { Tit! }\end{array}$ & 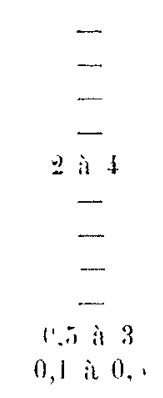 \\
\hline
\end{tabular}

Ci jont ergalement f'autres analyses d'anthracites que nous avons effectures stll dos arlhraciles rolsses et sur des anthraciles anglus. D)ans la fabricalion des électindes, los anthractos russer et anglass sont les plus recherchis, a cause de lent faible tenem en candres.

ANAIYSES D'AINIFIRACITES RUSSES (2)

\begin{tabular}{|c|c|c|c|c|c|c|c|c|c|c|c|c|}
\hline ANTHRACTTES & CARBONE & HYDROCEANE & OXYGENE & AZOTW & RTDRPE & $\begin{array}{c}\text { MATIERES } \\
\text { VOLATLLLS }\end{array}$ & $\begin{array}{l}\text { SOUFRL } \\
\text { ('TOT\L) }\end{array}$ & FTR & $\begin{array}{c}\text { PHOSPHORE } \\
\text { TUTs }\end{array}$ & SILILE & DEHISTEÉ & $\begin{array}{c}\text { POVIVOIR } \\
\text { CALIORIFIOULE }\end{array}$ \\
\hline$\backslash \cdots 1$ & 9517 & 1,11 & 2,60 & 0.7 .1 & 1,2 & $\delta, \pm$ & 0,2 & $(1,0)$ & Sianll & 0,217 & 1,39 & 8598 \\
\hline$\stackrel{2}{2}$ & 94.01 & $1, .30^{2}$ & 2,61 & $0,8 \pm$ & $1, \mathfrak{1}$ & 7,0 & 0,2 & $(1,1$ & Ninnl. & $0,3: 3 ;$ & 1,41 & 8610 \\
\hline$:$ & $94, \because \mathfrak{4}$ & 보 미를 & 2,61 & 0,83 & 1,1 & 8,5 & $0,: 3$ & $0, \ddot{2}$ & 0,006 & 0.303 & 1,44 & 86061 \\
\hline 1 & 80,2, & 3.91 & 4.97 & 1.83 & 1.5 & 8,7 & 0,3 & 0,3 & 0,004 & 0,093 & 1.48 & 8110 \\
\hline . & 90,29 & 3,60 & 3,21 & 1.97 & 1,8 & 7,6 & 0.3 & 0,2 & 0,008 & 0,621 & 1,60 & 8400 \\
\hline li & $11.0 \pm$ & 2,36 & 2,69 & 0,97 & 21 & 788 & $0, ?$ & 0.2 & 0,009 & $0, ., 100$ & 1,40 & $881 \%$ \\
\hline$i$ & 93,16 & 3,16 & 2,68 & 11 & 2,2 & 8,1 & 0,4 & 0,1 & 0,015 & 0,701 & 1,18 & 8970 \\
\hline 8 & 90,67 & $1,7 i$ & 2,97 & 172 & 2,1 & $9,1)$ & 0.8 & $0, \bar{i}$ & $0,0: 0$ & $0,80^{9}$ & 1,51 & 8190 \\
\hline !) & 䟚, & $: 2,28$ & 9,84 & 1,13 & 30 & $\bar{i}, \bar{i}$ & 0,0 & 0,5 & 0,041 & 1,012 & 1,47 & 8200 \\
\hline 10 & 91,12 & 1,42 & 2,45 & $1, \pi 1$ & $?, 9$ & $8, ;$ & 1,2 & 1,0 & $0.03 \mathrm{~J}$ & 1,1883 & 1,46 & 8811 \\
\hline 11 & 10,13 & 3,86 & 9,80 & 1,10 & 4,2 & 8,1 & 1,0 & 0.8 & 0,023 & $2,0,3 i$ & 1,19 & 8.390 \\
\hline 12 & $91,(69$ & 4,51 & 2,82 & 0,19 & 5,7 & 8.4 & 1,3 & 1.1 & 0030 & 2,065 & 1,57 & 8590 \\
\hline
\end{tabular}

ticulior des cellules parenchymateuses of des colyuscules arrondis ayant l'apparence de spores.

Lianthracite se rencontre sous formes de grander masses plus ou moine epaisses, dans los terrains de transituon,

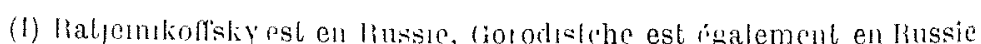
dans lo basan cin bonelz

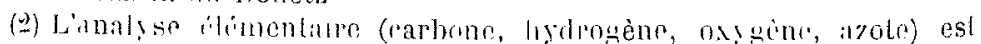

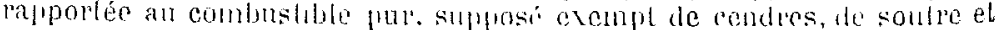
de phosphore. 
ANAIISES D'ANTIIRACIIES AINGIAIS (1)

\begin{tabular}{|c|c|c|c|c|c|c|c|c|c|c|c|c|}
\hline ANTHRALTTSS & CARBOND & HIDRORE: & OXYGene & AZOTS & CTHDRRE & $\begin{array}{l}\text { MATESRES } \\
\text { WULATLLS }\end{array}$ & $\begin{array}{l}\text { SOUPRE } \\
\text { (roT u.) }\end{array}$ & FIR & $\begin{array}{c}\text { PHOSPHORE } \\
\text { (тоти) }\end{array}$ & SLIER & DrNSTE & $\begin{array}{l}\text { POUWOIR } \\
\text { CALORIFLUE }\end{array}$ \\
\hline$X m 1$ & 90,21 & 4.17 & 4.70 & 0,83 & 1.0 & 8.6 & Nónt & 0.2 & vent & 0.3311 & 1,29 & 80010 \\
\hline 2 & 90,23 & 8,80 & $\pi, 01$ & 083 & 1,2 & 8,3 & Trnees & 0,3 & Néant & 0,307 & 1.32 & 83.61 \\
\hline 8 & 41,20 & $3, \pi$ & 4,27 & 0.70 & 8,3 & 8,2 & 0.2 & 01 & 0,049 & 0,859 & $1,4 i$ & $x^{2} \sin$ \\
\hline 4 & 01,50 & 3.89 & 4,47 & $0,-1$ & 3,2 & 8.0 & 0,4 & 0,5 & 0,000 & $0,10,1$ & $1,4$. & 8500 \\
\hline 5 & 02,13 & 3,99 & 3,50 & 0.99 & 3,3 & 8.5 & 0.8 & 19,3 & 0,008 & 1,171 & 1,38 & y $\mathrm{mol}$ \\
\hline i) & $(92,40$ & 3.28 & 3,58 & 0,7 & 3,1 & $\bar{i}, i$ & 0,6 & 0,1 & 001 & $1,130)$ & 1.34 & $8: 30$ \\
\hline 7 & 42,89 & 8,27 & 3,24 & 0,$6 ; 0$ & 9,7 & 4,2 & 0,5 & $(1,2 \geq$ & 00.89 & 0,27 & 1.11 & 8.450 \\
\hline 8 & 93,01 & 2.60 & 3,80 & 0,59 & 2,2 & 7.1 & 0,5 & 0,1 & 0,007 & 0.510 & 1, 但 & $x+40$ \\
\hline 4 & $13,3 \%$ & 2,54 & 8,46 & 0.63 & 1,4 & 8,5 & 0,3 & 0,2 & Viant & $0,3,86$ & $1,+\cdots$ & $\therefore 800$ \\
\hline 10 & 94.11 & 1,83 & 3,19 & $0,8 i$ & 1.5 & $x: 3$ & 4,4 & 0.8 & 0.001 & 0,168 & 1.37 & 1401 \\
\hline 11 & 94,60 & 1, ix & 2.81 & 0,82 & 1,8 & 8,8 & 0,2 & 0.2 & $0.00 \mathrm{~g}$ & $0,8.8 \pm$ & $1: 5$ & 8500 \\
\hline in & 11,7 & $1,-60$ & 2,74 & $1,0 \%$ & $\$ .4$ & $\mathrm{~s}, 0$ & $0, i$ & 0,8 & $0,0 \pm 3$ & 0180 & $1,4: 2$ & 8700 \\
\hline
\end{tabular}

b) Lomille. - La houlle ou chathon de lorre se frouvo à la lisière des terrains primitiss oul à l'motérieur de lours premiers plissoments. La surface des gi-ements houllers de la lerre peut être évaluce is environ un million do kilometres cartes, dont 20000 seulcinent en Euroje, bien que Thuropo fommisse a elle seule los trois quats do la production totale.

LAngletere viont au promer rang pour la production houllere, aver ses gisements du Cumberland et do la chame Penmine; les thats-Unis dhmerique occupent le deuxiemo mus, avec les gisements de la Virgmie, ie la Pensylvanie ef dil Mascachusetts; l'Allomagne le lroisieme rangr (gisoments de la Ruhr et de la llaute-Silesie) : ot la Franco le quatrieme rang A lni seul le bassin du Nord (Sens, Denain, Anzin) fournit la moltie du chiffre total de la production francasso, les bassins de St-Etienne, diais, du Creusot et de Blanzy venant ensuito.

La Belgiquo qui, copondant, possedo prestue toul lo has. sin ardennais, l'Autrohe-1Iongrie, la Ruscie, l'Espagne of llalle sont moins favoriséens fejendant la Russie poscèdo limportante gisemonts dan les valleses du bon of du Donet.

L'Afrique et l'Amerique dlu Sul ont des bassins encore insulfisamment déterminos lin Asio, il parnit existor dos gîtes de charbons faciloment accessibles dans l'est du Deccan, au Yun-Nan et dans les bascins de l'Amour et du Iloang-Ilo. L'Australio (Nouvelio-(ialles) possede egalement des gisements considérables.

Lorigino végétale de la louille ne fait plus actuellemont aucun doute. On attribua d'abord sa formation a l"enfouisment sur place des forcts et à leur carbonisation lente ì l'abri de l'air, car on avait trouvé des tiges dehout dans l'epaisseur des schistes et des gres houillers. Mais, plus tard, on conslata que le charbon de terre était formé d'éléments vógćtaux posés à plat los uns sur les autres, commo s'ils avatent eté accumulés par charriage, d'ou vint lidéc que les végétaux houillers avaient du souvont otre transportés par des courants d'cau assez loin de leur lien d'origine.

Comment les végétaux ont-ils été transformós on houille" L'hypothese la plus probahle, car elle esl vérifiéc par un cortain nombre de faits, est celle de la transformation dos lifférentes varićtés de collulose en houille sous linfluence microbienne.

On trouve beaucoup de houiles peuplées de bactériacces. En changeant la matière organique végétale on houlle, les bactériacées luj ont fait pordre les $4 / 5$ de sa substance

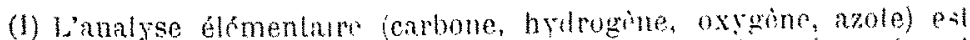

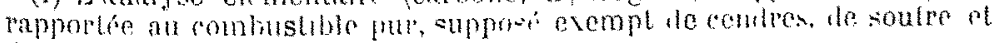
de phosphore. primulive, perte due a la formation de gaz tels que lantele "arlonique, lhydrogene of le méthane.

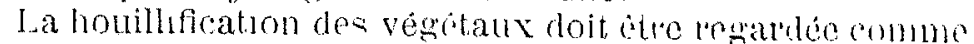

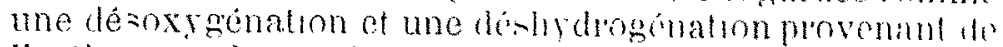
l'action simultanée de microcoques ot do hacillos tok que le

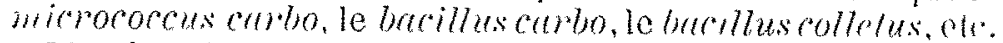

L'anthrarite ne présente que mroment les frares doinanisation, parce quo la dómposituon dos plantos est plus complite, of aussi, lans certains cas, paper quil whll

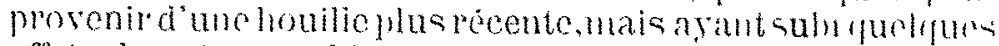
effets de métamoiphisine.

on divise ordinairement les houlles on cing lasso-

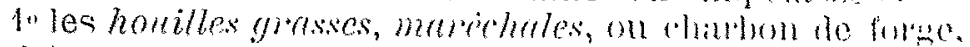
qui cprouvent au fou uno fusion pitcuso.

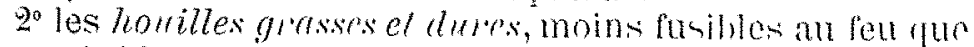
les précédentes.

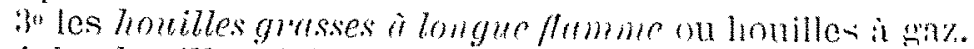

"fos houbles sches on mrigres, it longue flamme.

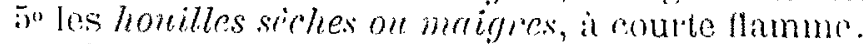

Les houilles do la promièc classo somllow seules conve. nables pom la falrication des électrodes, cal olles servut. surtout comme matiè agglutinante, en rason du ramollicsement qu'olles cprouvent au fru of do la srande quantiti: do matives soudromeuses ruellos renferment.

Nous avons effectué plusicurs analyses de ces houillos grassos et nous avons ohtenu les risultats consignó dians le tableau suivant: (1)

\begin{tabular}{|c|c|c|c|c|c|c|c|c|}
\hline $\begin{array}{l}\text { houlles } \\
\text { Jpasses }\end{array}$ & 总 & 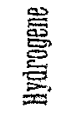 & 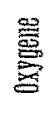 & 点 & 总 & 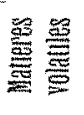 & 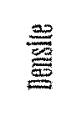 & 㲵 \\
\hline Nos I & $8 f 0$ & $\bar{i}, 0$ & 0,0 & \pm 0 & 111.6 & 38.6 & 1,2030 & X. (iil) \\
\hline & 812 & $0,:=$ & $\$, 0$ & $2, \pi$ & $12 .: 3$ & 3,3 & $1,2 \mathrm{~N}$ & $8 . \therefore(1)$ \\
\hline 3 & s6 4 & $\pi 1$ & $b i$ & 1.9 & 5,2 & 56,2 & $1,2 *$ & 7.0110 \\
\hline 4 & 88.27 & 5,8 & $d, 5$ & 19 & 4,7 & 五, & $1 . \pm 6$ & 8800 \\
\hline$\Rightarrow$ & 89.2 & 19 & 9,0 & $\$, ?$ & 4,5 & $2,5,5$ & $1, \pm 4$ & ถ. 260 \\
\hline 6 & 8,8 & $\cdot 5,4$ & $\pi, 4$ & 1,4 & 8,8 & 31,3 & 1,30 & $\vec{i} 9110$ \\
\hline 7 & 87.0 & 5.6 & 1,8 & 1,7 & 1,0 & 13,2 & 1.27 & 7.800 \\
\hline 8 & $80,1)$ & $5, \pi$ & 4,0 & 1,5 & 104 & 45,2 & $1,: 11$ & $\overrightarrow{7} 30(\mathrm{n})$ \\
\hline 9 & $8 k, 4$ & 5,6 & 8,7 & $\theta_{3}, 3$ & 5,1 & 95,8 & 1,20 & 8 专0 \\
\hline 10 & 87,7 & 5.0 & $\therefore, \pi$ & 1,6 & 7,2 & 24.1 & 1,27 & 5.3000 \\
\hline
\end{tabular}

c) Graphile. - - Ie exraphite, ou plombargine, so rencontre dans les gneiss, les micaschistes, los granitos et les ralcaires saccharoïdes des terrains primilifs. Il so présente souk forme de rognons, de lamelles, de reines et de lits de pou d'étendue.

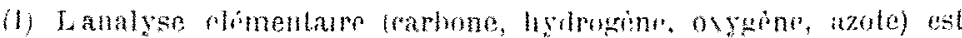

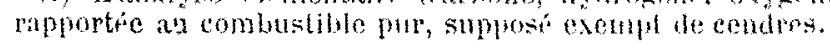


Lesplincipaux gisement de graphites sont ceux de Passau; Schwarzbach et Mugrau, en Bohôme; ceux de la Vloravie, de l'Autriche et de la Strrie. La Sibérie possède d'importantes mines de graphite, celles de Iéniséi, cellos des Monts Batougal découvertes en 1847 par l'ingénicur français Alibert. On trouve encore le graphite dans l'ile de Coylan. Il $y$ en a de puissants gisements dans l'Amérique du Nord, a Sturbridge (Massachussets), a Ficonderoza (New-Jersey) et à Fishkill (état de New-York) et en Californie. En France, it Chardonet (Hautes-Alpes), il y a un gisement de graphite d'une falble importanco.

Le graphite cristallise en lames hexagonales, il cst gris noirâtre, il a un éclat métallique et est doux el onctucux au toucher. Il tache les doigts et laisse sur le papier des traces d'un gris de plomb.

Sa densité est assez variable. Le graphite de Ceylan a une densité voisine de 2,25 ; celui de PAustralie, 2,66; celui de Bohème de 2,44 à 2,66 .

Après purification par la potasse en fusion el lavage aux acides, puis compressions et broyages alternos, afin d'éliminer les gaz occlus, on obtient toujours le chiffe 2,255

La quantilo de cendres renfermce dans le graphite est très variable et peut atteindre jusqu'à 60 pour 100 dans certains echantillons.

Ci-joint à litre d'exemple, cuelquos analyses de graphites effecluèes par Ch. Mène.

\begin{tabular}{|c|c|c|c|c|}
\hline Gin InIITES & CInnove & GENDNES & $\begin{array}{c}\text { MATHRES } \\
\text { VOLATILFS }\end{array}$ & DEX'SITE \\
\hline 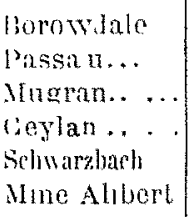 & $\begin{array}{l}91,55 \\
81,90 \\
91,05 \\
74,4 \\
88,05 \\
94,09\end{array}$ & $\begin{array}{r}7,35 \\
11,109 \\
4,85 \\
15,30 \\
10.90 \\
7,45\end{array}$ & $\begin{array}{l}1,10 \\
7,30 \\
1,10 \\
0,10 \\
1,0,7 \\
0,71\end{array}$ & $\begin{array}{l}2,3155 \\
2,3939 \\
2.1197 \\
2,3501 \\
2,1759 \\
2,1700\end{array}$ \\
\hline
\end{tabular}

\begin{tabular}{|c|c|c|c|c|c|c|c|c|}
\hline origine & $\begin{array}{l}\text { Matlèress } \\
\text { volatiless }\end{array}$ & Cendres & $\begin{array}{l}\text { Cartone } \\
\text { fixe }\end{array}$ & $\$ 10^{2}$ & $\mathrm{Al}^{2} 0^{3}$ & 0 & $\begin{array}{c}\text { CaO } \\
\text { et Mgo }\end{array}$ & Alcalls \\
\hline Gumberland & 1,10 & 7,35 & הימ, 91 & $0,0,0$ & 0,2833 & 0,190 & $0.0 \div 0$ & 0019 \\
\hline Passau.. & 7,30 & $11,1,2$ & 81.08 & $0,0,18$ & 0,356 & 0,068 & $(1,017$ & 0,029 \\
\hline Mugran. & 4,10 & 1,85 & 01,05 & 0,618 & 0,20 & 0,080 & 0,007 & 0,010 \\
\hline Seylan. & 5,90 & 2050 & 08,00 & $0,-, 013$ & 0,415 & 0,082 & 0,000 & 0,000 \\
\hline Austratie. & 2,15 & 72,10 & 25,75 & - & - & - & - & - \\
\hline Brésul. . & $2, \ldots$ & 20,30 & $i \pi, 15$ & 0.790 & 0,117 & 0,078 & 0,015 & 0,000 \\
\hline Brussin $\left(R^{n}\right)$ & $0, \pm 8$ & 7,72 & 18 & - & - & - & - & - \\
\hline Oural & 0,29 & 5,25 & 94,03 & 0612 & 0,297 & 01100 & 0,008 & 0,003 \\
\hline Sanadik. & 1,82 & 19,70 & 78,48 & $0,0,3$. & $0,2 \pi$ & 0,02 & $0,00 t_{5}$ & 0,012 \\
\hline
\end{tabular}

Ci-joint également quelques analyses effectuées par Kern cur du graphito de Stepand vsky (Sibéric) :

\begin{tabular}{|c|c|c|}
\hline GrAPIITIS & I & II \\
\hline Caphone ........... & 30,06 & 39,40 \\
\hline silee............. & 3,72 & 43,20 \\
\hline Sespuroxyte de fer & 402 & 3,05 \\
\hline Alumine ...... & 17,80 & 17,42 \\
\hline Lhind of magneste & $1, \pm 0$ & 1,106 \\
\hline Malieres volitiles... & 9,92 & 4,03 \\
\hline Soufre.. , ........ & 'Trinces & 0,04 \\
\hline
\end{tabular}

Comme on le voit, les graphites naturels sont très chargós en cendres ot demandent une purification à la fois mécanique of chimirue. On emploie dans ce but, soit les alcalis en fusion, solt les acides minéraux, tols que lacide fluorhydriquo et l'acide sulfurique.

(1 sllivre.)

Isidore BAY.

Ingénieur-Chimisle

\section{LE SERVICE D'ÉTUDE DEs GRANDES FORCES HYDRAULIQUES}

\section{PREMIERS RESULTATS}

Une ouvre dont l'mportance est capitale pour tous ccux qu1, dans notre pays, participent directement ou indrectemont i la mise en valeur de la houlle blanche, est en excellente vote de réalisation. 11 s'agit de la détermination méthodique et précise des facteurs de la puissance de nos chutes d'eau.

Pour aménager une section de rivière, il faut en connaître la pente et le débit I a détermination de la pente dépend d'un nivellement qui, tout en entraînant des frais, ne présente pas de grandes difficultés. Ma1s 1 n'en est pas de même du débit C'est l'élément de la puissance hydraulique le plus caché et le plus variable dont 1 imporie de mesurer exactement les différentes valeurs $C e$ ne sont pas seulement los débits extrêmes d'étrage ou de crues qu'on a besom de connaître, ce sont aussi les durées des divers débits intermédiaires au cours d'une année et les époques où ils se réalisient $c t$, comme c'est surtout en hydrologre qu'1l est vra1 que les années se suivent et ne se ressemblent pas, il faut pouvorr disposer d'observations portant sur un grand nombre d'annécs pour en dégager des moyennes à peu près exactes auxquelles on puisse comparer les résultats extrêmes correspondant aux années les plus sèches et les plus humides.

L'étude méthodique des grandes forces hydrauliques devait donc avoir pour base, d'abord l'établissement d'un certain nombre de stations permanentes de jaugeage dans les divers bassins de nos rivières, propres aux utılisations industrjelles. D'autre part, les débits des cours d'eau sont hés aux plures qui tombent dans leurs bassins par des lois complexes dépendant du nelief et de la nature géologique du sol. Il importe de déterminer ces lois pour chacun de nos bassins de montagne et de compléter amsi les indications des jaugeages directs, forcémont en nombre insuffisant.

Il faut donc étudier l'importance et la nature des précipitations dans chaque bassin, en même temps que par des mestres de planmétrue détaillées, l'on doit évaluer séparément. les surfaces du sol qui jouent un rôle différent dans l'alimentation des cours d'eau On se trouve ansi condult à s'occuper de pluviométrie et à planimétrer en détails chaque bassin.

La nécessité d'une telle étude était, depuis longtemps, reconnure. Le Congrès de la Houille blanche, tenu a Grenoble, en septembre I902, a pris, à cet égard, une résolution bien motivée. En effet, si l'on compare l'essor de l'industrie hydroélectrique dans les Alpes françaises, suisses ou italiennes, on constate que cette industrie, née en France, attx envirous do Grenoble, des premiers essais de Bergès, s'y est d'éveloppéc aussi rapidement qu'en aucune autre région, mais que, pat contre, la détermination exacte de la pussance hydraulique des cours d'eau est notablement moins avancée que chez nos voisins et que l'insuffisance des données sur lesquelles reposaient les premières entreprises a entraîné chez nous certains déboires. Ces considérations ont déterminé le Ministèro de l'Agriculture à organiser, par un décret en date du 25 mars I903, l'étude des questions qui se rapportent à l'évaluation des grandes forces hydrauliques en pays de montagne.

Ce Service a d'abord élé constitué dans les $\Lambda$ lpes pour itre ensute étendu aux Pyrénées ct, plus tard, à l'ensemble du territoire. Son organisation et sa direction ont été confiées aux deux ingénieurs les plus compétents qui se pussent trouver en ces questions et que nos lecteurs connaissent bien : MM. René TAVERNIER et R. DE IA BROSSE, ingénieurs en chof des Ponts et Chaussées.

Un premier rapport, que nous avons analysé très longuement dans le numéro de juillet 1906 , de La Honille Blmche, a fourni les renseignements préliminaires sur la façon dont 